\title{
C1QTNF6 promotes Oral squamous cell carcinoma tumorigenesis though enhancing proliferation and inhibiting apoptosis of OSCC cells
}

\section{Xiaobin Song}

Department of Oral and Maxillofacial Surgery,Qilu Hospital of Shandong University

\section{Longjie Li}

Department of Oral and Maxillofacial Surgery,Qilu Hospital of Shandong University

\section{Liang Shi}

Department of Oral and Maxillofacial Surgery, Qilu Hospital of Shandong University

\section{Xinyu Liu}

Department of Oral Medicine, Qilu Hospital of Shandong University

\section{Xun Qu}

Institute of Basic Medical Sciences, Qilu Hospital of Shandong University

\section{Fengcai Wei}

Department of Oral and Maxillofacial Surgery, Qilu Hospital of Shandong University https://orcid.org/0000-0003-1781-8661

Ketao Wang ( $\nabla$ wkt1972@sdu.edu.cn )

Department of Oral and Maxillofacial Surgery, Qilu Hospital of Shandong University https://orcid.org/0000-0001-6320-2767

\section{Primary research}

Keywords: Oral squamous cell carcinoma, C1QTNF6, Acute Phase Response signaling pathway

Posted Date: March 3rd, 2021

DOl: https://doi.org/10.21203/rs.3.rs-245470/v1

License: (c) (i) This work is licensed under a Creative Commons Attribution 4.0 International License. Read Full License 


\section{Abstract}

\section{Background}

C1QTNF6 (CTRP6), a member of the CTRP family, has been recently implied to play a role in tumorigenesis. However, the expression status and the role of C1QTNF6 in oral squamous cell carcinoma (OSCC) remains unclear.

\section{Methods}

Immunohistochemistry of OSCC tissue and data from TCGA both implied that C1QTNF6 was closely related to OSCC. We constructed lentivirus to knockdown C1QTNF6 in CAL27 cells and SCC-9 cells. Then the change of C1QTNF6 mRNA expression was detected with qRT-PCR, and the Western blot analysis was performed to detect changes in protein expression. Furthermore, Cell Cycle Analysis and Cell apoptosis analysis was measured. 4-week-old female BALB/c nude mice were purchased to observe the In vivo tumorigenicity. Finally, Pathway Analysis was performed.

\section{Results}

In this study, we found that C1QTNF6 was overexpressed in OSCC tissues and cell lines, and the cellular proliferation was significantly decreased in C1QTNF6 knockdown OSCC cells. Knockdown of C1QTNF6 resulted in cell cycle arrest at the G2/M phase and enhanced apoptosis in OSCC cell lines. Further assays showed that C1QTNF6 silencing inhibits tumor growth of OSCC in vivo. Moreover, microarray analysis revealed that C1QTNF6 silencing results in significant alteration of many genes. Ingenuity Pathway Analysis (IPA) revealed that the Acute Phase Response signaling pathway was significantly activated following C1QTNF6 silencing.

\section{Conclusions}

These results suggested that C1QTNF6 play a promoting role in OSCC tumorigenesis, which may be a promising therapeutic target for OSCC treatment.

\section{Background}

Oral squamous cell carcinoma (OSCC) is the most common epithelial malignancy in the oral cavity, which accounts for almost $90 \%$ of all oral malignancies[1]. Despite the improvements in surgical and other therapeutic strategies, the 5 -year survival rate of OSCC patients is still disappointing[2, 3]. So far, limited information about the driving forces and molecular pathways in OSCC tumor formation is available, and the molecular events have not been defined precisely. Thus, a better understanding of the molecular carcinogenesis of OSCC may provide new insights on the development of novel diagnostic and therapeutic strategies, and the improvement of OSCC patients' prognosis. 
C1q/tumor necrosis factor-related proteins (CTRPs/C1QTNF), including 16 members, consisting of four different domains: an N-terminal signal peptide, a collagenous-like domain, a short variable region, and a C-terminal globular domain[4]. CTRPs share a common structural domain with adiponectin[5]. C1QTNF6 (CTRP6) is the sixth member of the CTRP family and is widely expressed in many tissues of rodents and primates, including adipose tissue, brain, spleen, lung, liver, muscle, ovary, and so on[6-10], and each CTRP has a unique tissue expression profile[11]. C1QTNF6 is secreted in serum-forming oligomeric forms[12] and is involved in diverse physiological processes ranging from metabolism, to host defense and organogenesis[13]. It has been reported that C1QTNF6 can stimulate fatty acid oxidation via activation of AMPK[14]. By generating C1QTNF6-/- mice, it has been demonstrated that C1QTNF6 is a novel regulator of the complement alternative pathway, and can be used to treat rheumatoid arthritis in clinical[15]. One study showed that CTRP4 promoted tumor survival through upregulation of IL-6 and TNF-alpha[16]. Recent researches suggested that CTRP6 may play a role in carcinogenesis and cancer progression. Recent studies showed that C1QTNF6 was overexpressed in clear cell renal cell carcinoma and elevated C1QTNF6 expression correlated with clinical progression[17].It has been confirmed that C1QTNF6 is involved in promoting the proliferation, migration, and in reducing the apoptosis of gastric carcinoma cells and NSCLC $[18,19]$. A study showed that inhibition of C1QTNF6 induced G2-M cell cycle arrest and cell apoptosis[18]. And it has been reported that C1QTNF6-related signaling pathways activated in ccRCC were mainly enriched in DNA replication, Cell Cycle, EMT, and angiogenesis signaling pathway[17]. In particular, the overexpression of C1QTNF6 has been shown in hepatocellular carcinoma tissues and many HCC cell lines, and promotes neovascularization in transplanted HCC cells[20]. These researches suggested a potential regulatory role of C1QTNF6 in tumorigenesis, which makes great sense to further study the function and mechanism of C1QTNF6 in other cancers.

In this study, we confirmed the high expression of C1QTNF6 in OSCC tissues by immunohistochemical analysis and cell lines by RT-PCR. Then we analyzed The Cancer Genome Atlas (TCGA) and found that C1QTNF6 might be a potential tumor-associated regulator involved in carcinogenesis of OSCC. Furthermore, the C1QTNF6 knockdown was performed in OSCC cell lines to explore the pathological relevance between C1QTNF6 and OSCC. We found that C1QTNF6 promoted the proliferation and suppressed the apoptosis of OSCC cells in vitro. Moreover, the xenograft tumor growth was inhibited by C1QTNF6 silence in vivo. In addition, the signal pathway was analyzed to explore the possible molecular mechanism underlying the regulatory function of C1QTNF6 in OSCC by IPA. The data showed that the Acute Phase Response signal pathway might play a role in C1QTNF6-mediated promotion of OSCC

\section{Materials And Method}

\section{Cell lines}

CAL27 cells and SCC-9 cells『the head and neck squamous cell carcinoma cell line『were cultured in DMEM (Gibco, USA) supplemented with 10\% fetal calf serum (Thermo Fisher Scientific, USA), $100 \mu \mathrm{g} / \mathrm{ml}$ streptomycin (Gibco, USA), and 100U/ml penicillin (Gibco, USA) at $37^{\circ} \mathrm{C}$ with $5 \% \mathrm{CO} 2$. 
For expression analysis, we selected RNAseq and RNAseqV2 paired sample data for analysis. The raw sequencing data and pathological information were downloaded from the TCGA database (https://cancergenome.nih.gov/). For Head and Neck squamous cell carcinoma (HNSCC), there were 528 samples with available data in the TCGA database, including 520 RNAseqV2 samples, and 40 pairs with paired sample data and pathological information. Our expression profile analysis was based on these 40 paired samples of RNAseqV2 data. The Trimmed Mean of M-values (TMM) method was applied to data standardization. To avoid errors caused by inappropriate sample grouping, BCV『biological coefficient of variation $₫$ was observed for quality control.

Firstly, we estimated the dispersion of multiple pairs of samples and then used a general linear model to estimate whether there is a difference in genes between different groups. Genes with a statistical test $P$ value less than 0.05 are considered differentially expressed genes. The fold changes (FCs) in the expression were calculated and differentially expressed DEGs with log2|FC| $\geq 1.0$ (Cancerrmal) were considered to be significant, remaining genes are filtered. Besides, several other criteria were adopted to filter genes: the genes which had been reported have functional and clinical relation in HNSCC; genes of multiple transmembrane proteins; gene annotation is not clear (such as open reading frame); genes reported in more than 100 articles in Pubmed. The final gene list was randomly concentrated, and C1QTNF6 was selected for further experimental research.

\section{Lentivirus Construction and lentiviral transfection of CAL27 cells and SCC-9 cells}

C1QTNF6-targeting short hairpin RNA (shRNA) oligonucleotides sequences $\ T$ GTGTGAGATCCCTATGGT $\$ were designed and were synthesized and cloned into the pGV115-GFP vector by GeneChem Corporation (Shanghai, China). The sequence of scrambled shRNA (TCTCCGAACGTGTCACGT) used as the negative control (NC) was also inserted into the pGV115-GFP vector. Lentivirus transfection was performed according to the manufacturer's recommended protocol. The CAL27 cells and SCC-9 cells were seeded into a six-well plate $\left(\sim 1 \times 10^{5}\right.$ cells per well) and incubated at $37^{\circ} \mathrm{C}$ with $5 \% \mathrm{CO}_{2}$ until they reached $\sim 70 \%$ confluence, then lentivirus was added as $\mathrm{MOI} 1$. After $72 \mathrm{~h}$, cells were observed under a fluorescence microscope and collected for the following experiments.

\section{RNA extraction and quantitative real-time PCR (qRT-PCR)}

Total RNA was isolated from cells using TRIzol (Invitrogen, USA) according to the manufacturer's instructions. Reverse transcription was performed to synthesize cDNAs using M-MLV reverse transcriptase (Promega) and OligodT primers (Sangon, Shanghai). C1QTNF6 mRNA expression was detected with quantitative real-time PCR using SYBR Master Mixture (Takara, Japan) on a Real-Time PCR machine TP800 (Takara). The cycling parameters were $95^{\circ} \mathrm{C}$ for a 30 s hot start followed by 45 cycles of $95^{\circ} \mathrm{C}$ for $5 \mathrm{~s}$ and $60^{\circ} \mathrm{C}$ for $30 \mathrm{~s}$. Primers used here were as follows: GAPDH for: $5^{\prime}-$

TGACTTCAACAGCGACACCCA -3', GAPDH reverse:5'-CACCCTGTTGCTGTAGCCAAA -3', C1QTNF6 for: 5'- 
GAAAGGGTCTTTGTGAACCTTGA -3', and C1QTNF6 reverse: 5'- CTGCGCGTACAGGATGACAG -3'. The relative mRNA expression was calculated using the $2^{-\triangle \triangle C t}$ method.

\section{Western blot analysis}

Cell samples were harvested, washed twice with PBS, and Lysed with pre-cooled Lysis Buffer. The protein concentration was determined by BCA Protein Assay Kit (Pierce). Each sample protein concentration is adjusted to $2 \mu \mathrm{g} / \mu \mathrm{L}$, and saved at $-80^{\circ} \mathrm{C}$ for later use. The total protein was separated on $10 \%$ sodium dodecyl sulfate polyacrylamide gel electrophoresis (SDS-PAGE) and transferred to polyvinylidene fluoride (PVDF) membranes at $4^{\circ} \mathrm{C}(300 \mathrm{~mA}$ for $150 \mathrm{~min})$. The PVDF membranes were blocked in $5 \%$ skim milk in TBST for $1 \mathrm{~h}$ and incubated overnight with primary antibodies at $4^{\circ} \mathrm{C}$. After washing three times in TBST, the membranes were incubated in secondary antibody coupled to HRP before visualization.

\section{Immunohistochemistry}

13 paired cancer and paracancerous tissue samples were collected from OSCC patients in Qilu hospital of Shandong University, both preoperative and postoperative pathology of which suggest oral squamous cell carcinoma. All patients gave informed consent before the tissue was collected. The research had obtained the approval of Ethics Committee on Scientific Research of Shandong University Qilu Hospital and the approval number is KYLL-2017-544. OSCC tissues and normal tissues were immunostained for C1QTNF6 expression using a C1QTNF6 antibody (Invitrogen, USA) at a 1:100 dilution for a 1-h incubation according to the supplier's instructions. The secondary antibody used was a goat anti-rabbit IgG incubated for $1 \mathrm{~h}$ at $37^{\circ} \mathrm{C}$. Images were obtained at the optical facility.

\section{Cell Cycle Analysis by Flow Cytometry}

Lentivirus-transfected cells were seeded in 6-cm dishes for culture. When the cells achieved approximately $80 \%$ confluence, they were trypsinized, washed twice in PBS, and fixed with pre-chilled $70 \%$ ethanol for at least $1 \mathrm{~h}$ at $4{ }^{\circ} \mathrm{C}$. Then the cells were washed with PBS and stained with PI mixture (40×PI stock $(2 \mathrm{mg} / \mathrm{ml}), 100 \times \mathrm{RNase}$ stock $(10 \mathrm{mg} / \mathrm{ml})$ and $1 \times$ PBS buffer at a dilution of 25:10:1,000) for $45 \mathrm{~min}$ at $37^{\circ} \mathrm{C}$. The cell cycle results were measured by Guava easyCyte HT (Millipore, USA), and all experiments were performed in triplicate.

\section{Annexin V Apoptosis Assay}

Cell apoptosis analysis was measured using eBioscience ${ }^{\mathrm{TM}}$ Annexin V Apoptosis Detection Kit APC according to the manufacturer's instructions. Briefly, Lentivirus-transfected cells were trypsinized, washed twice in pre-chilled D-hank's, and resuspended in binding buffer. Then $5 \mu$ of annexin V-APC was added into $100 \mu \mathrm{l}$ of the cell suspension and incubated at room temperature in the dark for $15 \mathrm{~min}$. Samples were analyzed by Guava easyCyte HT (Millipore, USA).

In vivo tumorigenicity 
Twenty 4-week-old female BALB/c nude mice were purchased from the Shanghai Institute for Biological Sciences (Shanghai, China). Twenty BALB/c nude mice were randomly divided into two groups. The lentivirus-transfected cells $\left(1 \times 10^{7}\right)$ were inoculated into the right side of the axillary of nude mice subcutaneously as previously described. The mice were observed every 5 days after anesthesia by intraperitoneal injection of $0.7 \%$ pentobarbital sodium according to the dosage of $10 \mu \mathrm{L}$ per gram of body weight. The length and width of tumors were measured using calipers, and the volume of tumors was calculated using the equation $\left(L \times W^{2}\right) / 2$. On day 53 , tumor growth was monitored by luciferase activity detection using the IVIS imaging system. Meanwhile, the mice were euthanized by injection of an overdose of $2 \%$ pentobarbital, after which cervical dislocation was performed to confirm death, and the tumors were removed and weighed.

\section{Pathway Analysis (IPA)}

Firstly, a signal histogram was made to show the signal intensity distribution of all chip probes, and the average Z-score value of all samples in the same signal intensity interval is less than 2 . Relative Signal Box Plot showed the medians of all samples, and the Z-score value of the medians was less than 2. Correlation Analysis was demonstrated by the Pearson correlation coefficient distribution chart, which indicated that the correlation coefficients within both groups were all greater than 0.99 . The principal component analysis was then conducted. All the works above confirmed that the data owned fine reliability, repeatability, also significant differences between groups, so it met the criteria for continued analysis. The lowest $20 \%$ of the signal intensity of the probe set was filtered out as background noise. Secondly, we used the coefficient of variation method to calculate the coefficient of variation of the same probe in the same sample, and filtered out probes with a coefficient of variation greater than $25 \%$ in both groups. Finally, we got data of 39,287 probes.

We used a linear model based on empirical Bayes distribution to calculate the significant difference level P-value and used the Benjamini-Hochberg method to correct the significant difference level. The screening criteria for significantly different genes are |Fold Change| $>1.5$ and FDR $<0.05$. Hierarchical Clustering was proceeded. The clustering algorithm classifies samples and variables in two dimensions.

We used the Ingenuity Pathways Analysis (IPA, Ingenuity systems, Inc., Redwood City, CA, www.ingenuity.com) tool to examine biological functions and disease as well as functional relationships between genes and gene networks.

\section{Results}

\section{C1QTNF6 is high expressed in OSCC tissue and cell lines}

We downloaded and analyzed 40 paired data from The Cancer Genome Atlas (TCGA) and found that C1QTNF6 might be a potential tumor-associated regulator involved in the carcinogenesis of OSCC (Fig. 1a). Furthermore, we collected 13 paired OSCC tissue and normal tissue and discovered the high expression of C1QTNF6 in OSCC tissues by RT-PCR. 
Then we examined the expression of C1QTNF6 in human OSCC tissue by immunohistochemical analysis and cell lines Cal-27, SCC-9 by RT-PCR. Compared with normal tissues, the expression of C1QTNF6 was higher in the OSCC tissues (Fig. 1b\&c). The high levels of C1QTNF6 mRNA in Cal-27 and SCC-9 were observed (Fig. 1d).

\section{The efficiency of C1QTNF6 knockdown by Lentiviral-Based shRNA in human oral squamous cell carcinoma cell lines}

To explore the biological role of C1QTNF6 in OSCC tumorigenesis, the lentiviral-based shRNA was constructed to knockdown C1QTNF6 in human OSCC cell lines cal-27 and SCC-9. More than $90 \%$ of siRNA-transfected cells expressed GFP under a fluorescent microscope at 48h after lentivirus infection, which suggested a high infection efficiency (data not shown). The Efficiency of the C1QTNF6 knockdown was confirmed by qRT-PCR and western blot. C1QTNF6 mRNA levels were significantly reduced in both cell lines after C1QTNF6-shRNA transfection, showing approximately 60-80\% knockdown efficiency (Fig. 2a). Western blot analysis showed the dramatically decreased C1QTNF6 protein expression in C1QTNF6shRNA transfected cells compared to that in Ctrl-shRNA transfected cells (Fig. 2b).

\section{Cell proliferation was inhibited by C1QTNF6 knockdown in human oral squamous cell carcinoma cell lines}

Sustained proliferation is a hallmark of cancer cells. To detect the effect of the C1QTNF6 gene on cell proliferation of OSCC cell lines, we performed two different assays to evaluate cell proliferation. For Celigo assay, the viable cells infected with LV-C1QTNF6-shRNA or LV-Ctrl-shRNA were counted every day for five consecutive days by Celigo Cytometer. Silencing of C1QTNF6 lowered the cell growth curve from day 3 to day 5 in Cal-27(Fig. 3a) and SCC-9 cells (Fig. 3b). And the inhibition of cell growth by C1QTNF6 silence was more pronounced over time. Additionally, an MTT assay was carried out to detect cell viability. We found that optical density at $490 \mathrm{~nm}$ increased by 3.3-fold in Cal-27 cells treated with CtrlshRNA and 1.1-fold in those treated with C1QTNF6-shRNA at the 4 days after transfection in Cal-27 (Fig. 3c). Similar results were observed in SCC-9 cells (Fig. 3d). Together, the data indicated that the knockdown of C1QTNF6 significantly inhibited cell proliferation in human oral squamous cell carcinoma cell lines.

\section{C1QTNF6 knockdown induced cell cycle arrest and apoptosis of human oral squamous cell carcinoma cell lines}

To find out whether the promoting effect of C1QTNF6 on the proliferation of OSCC cell lines was achieved by promoting cell cycle stability or inhibiting apoptosis, we used PI staining to measure cell cycle distribution and Annexin-V staining to assess apoptosis in Cal-27 and SCC-9 cells modified by CtrlshRNA or C1QTNF6-shRNA. As shown in Fig. 4a\&b, Ctrl-shRNA transfected Cal-27 cells had the following cell cycle distribution: G0/G1 55.47\%, S 26.28\%, G2/M 18.25\%; knockdown of C1QTNF6 significantly reduced the fraction of cells in the $S$ phase, and significantly increased the fraction in the G2/M phase, with the following cell cycle distribution: G0/G1 62.77\%, S 14.94\%, G2/M 22.92\% (all P< 0.05). These 
data demonstrate cell cycle progression through the G2/M phase was hindered in Cal-27 cells after C1QTNF6 silencing. Similar results were shown in SCC-9 cells. Furthermore, the apoptosis of Cal-27 cells and SCC-9 cells were significantly increased by C1QTNF6 knockdown (Fig. 4c\&d). These results suggested that C1QTNF6 silencing impeded cell cycle progression and promoted apoptosis in OSCC cell lines.

\section{C1QTNF6 promote human oral squamous cell carcinoma cell growth in vivo}

To extend the in vitro observations, we investigated the effects of C1QTNF6 knockdown on tumor growth in vivo. Cal-27 cells were used for subsequent experiments. Cal-27 cells that were stably transfected with either C1QTNF6-shRNA or Ctrl-shRNA lentivirus were injected into nude mice, and the tumor growth was monitored. Tumors formed by C1QTNF6-silenced Cal-27 cells were much smaller during the experiment period than the control tumor (Fig. 5a\&b). During necropsy, tumors were excised and weighed. Ctrl-shRNA tumors weighed on an average of $1300 \mathrm{mg}$ whereas C1QTNF6-shRNA tumors remained at $400 \mathrm{mg}$ (Fig. 5c). Collectively, these results emphasize the promoting role of C1QTNF6 in cancer progression in vivo.

\section{C1QTNF6 knockdown may inhibit the tumorigenesis of OSCC through Acute Phase Response signaling pathway by targeting ID1, BBC3 and DDIT3}

The above results suggest that C1QTNF6 played a critical role in the development of OSCC. To elucidate the molecular mechanisms by which C1QTNF6 promotes malignancy of the OSCC cells, microarray analysis was used to compare gene expression between cells infected with Ctrl-shRNA and C1QTNF6shRNA lentivirus. The data revealed that the expression of 1628 genes was identified which showing significant differentiation ( $P<0.05$ and |Fold Change| $>1.5$ ), including 943 upregulated genes and 685 downregulated genes. The heat map showed hierarchical clustering analysis of differentially expressed genes which revealed that OSCC cells from three samples shared a quite similar gene expression patterns following C1QTNF6 knockdown (Fig. 6a). Next, canonical pathway analysis revealed that the Acute Phase Response signaling pathway was significantly activated following C1QTNF6 knockdown according to the expression trend of molecules detected in the experimental results (Fig. 6b). Disease and function prediction analysis results showed that Cancer is significantly activated (Fig. 6c). Connections among C1QTNF6 and several closely related molecules were built based on the existing findings (Fig. $6 \mathrm{~d})$. Western blot verified the expression variation of several molecules in the network above区especially ID1, BBC3, and DDIT3, which are target genes of TNF (Fig. 6e).

\section{Discussion}

C1QTNF6 is a crucial molecular mediator connecting inflammation, fibrosis, and metabolism[14], and it has been implied in the involvement of tumor development. The role of C1QTNF6 in tumor cell survival and cell cycle has been investigated in other cancer $[18,20]$. However, the study focusing on the relationship between C1QTNF6 and OSCC tumor development remains limited. In the present study, we reported high levels of C1QTNF6 expression in OSCC tissue and cell lines. In addition, the C1QTNF6 knockdown delayed the proliferation of OSCC cells and induced cell apoptosis in vitro. Inconsistent with 
our observation, one study showed that the expression of C1QTNF6 in human gastric carcinoma tissues was higher than that in normal gastric tissue, and C1QTNF6 silence decreased the growth and resulted in G2-M cell cycle arrest in gastric carcinoma cells[18]. And it has been reported that C1QTNF6-related signaling pathways activated in clear cell renal cell carcinoma were mainly enriched in DNA replication, Cell Cycle, EMT, and angiogenesis signaling pathway[17]. In line with this observation, we found that C1QTNF6 interference increased the percentage of cells at the G2-M phase in human OSCC cell lines. To extend the in vitro observations, we investigated the effect of C1QTNF6 on tumor growth in vivo. We found a retarded tumor growth in mice inoculated with C1QTNF6-silenced cells. This result further emphasized the promoting role of C1QTNF6 in OSCC progression, which may stimulate tumor proliferation.

To date, the molecular mechanism underlying the function of C1QTNF6 in OSCC remains unclear. As shown above, we have demonstrated the important role of C1QTNF6 in OSCC development and progression. To elucidate the molecular mechanisms by which C1QTNF6 promotes malignancy of OSCC, microarray analysis was performed. Hundreds of genes showed significantly differential expression between C1QTNF6-shRNA and Ctrl-shRNA transfected cells. According to the data of Ingenuity Pathway Analysis (IPA), the Acute Phase Response was the top-activated signaling pathway following C1QTNF6 silencing. This signaling pathway is an important component of anticancer responses among multicellular organisms[21]. The acute phase response is a nonspecific physiological and biochemical reaction to tissue damage, infection, inflammation, and neoplasia during which the synthesis of several plasma proteins is increased (positive acute-phase proteins) or decreased (negative acute-phase proteins)[22]. The acute phase response is not diagnostic for any particular kind of disease but occurs as a response to several pathological conditions and diseases, including bacterial infections, sepsis, surgery, trauma, myocardial infarction, inflammatory diseases, and cancer[23]. Besides, other pathways showed altered activation status after C1QTNF6 knockdown, such as TREM1 signaling, IL-6 signaling, and Tolllike Receptor signaling, can induce tumor cell apoptosis or activate an anti-tumor immune response, hence be implicated in the development of variant cancers[24-26]. What's more, western blot assay showed that ID1 was significantly down-regulated by C1QTNF6 knockdown. In our previous study, we have demonstrated that the over-expression of ID1 effectively promoted the carcinogenesis of OSCC[12, 13]. ID1 is one of the inhibitors of DNA binding (Id) proteins[27]. During development, the Id proteins play a key role in the regulation of cell-cycle progression and cell differentiation by modulating different cellcycle regulators both by direct and indirect mechanisms[28]. ID1 enhanced cell proliferation, colony formation, and tumor growth by regulating the cell cycle in lung cancer[29]. Based on the studies above, we speculated that knockdown of C1QTNF6 caused the low expression of ID1, which further led to cell cycle arrest in OSCC.

BH3-only protein $\mathrm{BBC}$ (BCL-2-binding component 3) belongs to the $\mathrm{Bcl}-2$ family and is a strongly proapoptotic gene that is subject to transcriptional regulation by multiple cell death-signaling pathways[30]. It has become obvious that BBC3 plays a role in p53 induced apoptotic pathways and can also function independently of p53. DDIT3, also known as C/EBP homologous protein (CHOP) or growth arrest and DNA damage-inducible gene 153 (GADD153), is a member of the CCAAT/enhancer-binding 
protein (C/EBP) family ${ }^{[31]}$. DDIT3(DNA-damage inducible transcript 3) promoted mice ovarian cells apoptosis via ER stress activation, and knockdown of DDIT3 suppressed cell apoptosis[32]. DDIT3 is an important transcription factor of apoptosis under ER stress and the DDIT3-mediated apoptosis pathway is one of the most dominant pathways in the apoptosis process[33]. Over-expression of CHOP has been reported to lead to cell cycle arrest and/or apoptosis, and CHOP has been shown to regulate numerous pro- and apoptotic genes as a transcriptional factor[34]. High levels of BBC3, DDIT3, and obvious cell apoptosis after C1QTNF6 knockdown were observed in this study. Thus, we tend to believe that knockdown of C1QTNF6 induced the occurrence of endoplasmic reticulum stress and the overexpression of BBC3 and DDIT3 which leads to apoptosis of OSCC cell lines in vitro. Our results of microarray analysis fit well with the known effects of the above-mentioned signaling pathways and genes on tumorigenesis, suggesting that the promotion of tumor development by C1QTNF6 might be mediated through regulating the Acute Phase Response signaling and ID1, BBC3, DDIT3 gene expression.

\section{Conclusions}

There is a balance between the proliferation and apoptosis of tumor cells, and how to regulate this balance determines the development and progression of the tumor. In summary, we speculated that the C1QTNF6 knockdown led to complex changes in OSCC, which might produce an anti-cancer effect through acute phase response both in vivo and in vitro. The microarray analysis suggested that target genes, including but not limited to ID1, BBC3, and DDIT3, the changes in the expression level of which could cause cell cycle arrest and promote apoptosis of OSCC. Our study provided the possible molecular mechanisms underlying C1QTNF6-mediated promotion of OSCC tumorigenesis, which need to be further studied, especially cell cycle factor, apoptosis protein, and ER stress. Our findings not only provide a novel target gene for OSCC therapy but also add new evidence supporting the relationship between C1QTNF6 and tumorigenesis.

\section{Declarations}

\section{Ethics approval and consent to participate}

The present study was approved by The Ethics Committee of Qilu Hospital of Shandong University (Jinan, China) and informed consent was provided by all the patients prior to the start of the study.

\section{Consent for publication}

Not applicable.

\section{Availability of data and materials}

The datasets used or analysed during the current study are available from the corresponding author on reasonable request. 
Competing interests

The authors declare that they have no competing interests.

Funding

This work was in part supported by Key Technology Research and Development Program of Shandong, China (grant No.2017GSF221008 and No.2019GSF107016).

\section{Authors' contributions}

XS wrote the manuscript, and performed the experiments. LL analyzed and interpreted the patients' data, and helped with the writing of the manuscript. All authors read and approved the final manuscript.

\section{Acknowledgements}

The authors thank all contributors of the present study.

\section{References}

1. Warnakulasuriya S: Global epidemiology of oral and oropharyngeal cancer. Oral oncology 2009, 45(4-5):309-316.

2. Kamarajan P, Garcia-Pardo A, D'Silva NJ, Kapila YL: The CS1 segment of fibronectin is involved in human OSCC pathogenesis by mediating OSCC cell spreading, migration, and invasion. $B M C$ cancer 2010, 10:330.

3. Wu CH, Wu TY, Li CC, Lui MT, Chang KW, Kao SY: Impact of diabetes mellitus on the prognosis of patients with oral squamous cell carcinoma: a retrospective cohort study. Annals of surgical oncology 2010, 17(8):2175-2183.

4. Klonisch T, Glogowska A, Thanasupawat T, Burg M, Krcek J, Pitz M, Jaggupilli A, Chelikani P, Wong GW, Hombach-Klonisch S: Structural commonality of C1q TNF-related proteins and their potential to activate relaxin/insulin-like family peptide receptor 1 signalling pathways in cancer cells. British journal of pharmacology 2017, 174(10):1025-1033.

5. Wong GW, Wang J, Hug C, Tsao TS, Lodish HF: A family of Acrp30/adiponectin structural and functional paralogs. Proceedings of the National Academy of Sciences of the United States of America 2004, 101(28):10302-10307.

6. Akiyama H, Furukawa S, Wakisaka S, Maeda T: CTRP3/cartducin promotes proliferation and migration of endothelial cells. Molecular and cellular biochemistry 2007, 304(1-2):243-248.

7. Park SY, Choi JH, Ryu HS, Pak YK, Park KS, Lee HK, Lee W: C1q tumor necrosis factor alpha-related protein isoform 5 is increased in mitochondrial DNA-depleted myocytes and activates AMP-activated protein kinase. The Journal of biological chemistry 2009, 284(41):27780-27789. 
8. Peterson JM, Wei Z, Wong GW: C1q/TNF-related protein-3 (CTRP3), a novel adipokine that regulates hepatic glucose output. The Journal of biological chemistry 2010, 285(51):39691-39701.

9. Chavali VR, Khan NW, Cukras CA, Bartsch DU, Jablonski MM, Ayyagari R: A CTRP5 gene S163R mutation knock-in mouse model for late-onset retinal degeneration. Human molecular genetics 2011, 20(10):2000-2014.

10. Hofmann C, Chen N, Obermeier F, Paul G, Büchler C, Kopp A, Falk W, Schäffler A: C1q/TNF-related protein-3 (CTRP-3) is secreted by visceral adipose tissue and exerts antiinflammatory and antifibrotic effects in primary human colonic fibroblasts. Inflammatory bowel diseases 2011, 17(12):2462-2471.

11. Seldin MM, Tan SY, Wong GW: Metabolic function of the CTRP family of hormones. Reviews in endocrine \& metabolic disorders 2014, 15(2):111-123.

12. Ghai R, Waters P, Roumenina LT, Gadjeva M, Kojouharova MS, Reid KB, Sim RB, Kishore U: C1q and its growing family. Immunobiology 2007, 212(4-5):253-266.

13. Kim MJ, Lee W, Park EJ, Park SY: C1qTNF-related protein-6 increases the expression of interleukin-10 in macrophages. Molecules and cells 2010, 30(1):59-64.

14. Lee W, Kim MJ, Park EJ, Choi YJ, Park SY: C1qTNF-related protein-6 mediates fatty acid oxidation via the activation of the AMP-activated protein kinase. FEBS letters 2010, 584(5):968-972.

15. Murayama MA, Kakuta S, Inoue A, Umeda N, Yonezawa T, Maruhashi T, Tateishi K, Ishigame H, Yabe $\mathrm{R}$, Ikeda $\mathrm{S}$ et al: CTRP6 is an endogenous complement regulator that can effectively treat induced arthritis. Nature communications 2015, 6:8483.

16. Li Q, Wang L, Tan W, Peng Z, Luo Y, Zhang Y, Zhang G, Na D, Jin P, Shi T et al: Identification of C1qTNF-related protein 4 as a potential cytokine that stimulates the STAT3 and NF-KB pathways and promotes cell survival in human cancer cells. Cancer letters 2011, 308(2):203-214.

17. Lin W, Chen X, Chen T, Liu J, Ye Y, Chen L, Qiu X, Chia-Hsien Cheng J, Zhang L, Wu J et al: C1QTNF6 as a Novel Diagnostic and Prognostic Biomarker for Clear Cell Renal Cell Carcinoma. DNA and cell biology 2020, 39(6):1000-1011.

18. Qu HX, Cui L, Meng XY, Wang ZJ, Cui YX, Yu YP, Wang D, Jiang XJ: C1QTNF6 is overexpressed in gastric carcinoma and contributes to the proliferation and migration of gastric carcinoma cells. International journal of molecular medicine 2019, 43(1):621-629.

19. Zhang W, Feng G: C1QTNF6 regulates cell proliferation and apoptosis of NSCLC in vitro and in vivo. Bioscience reports 2020.

20. Takeuchi T, Adachi Y, Nagayama T: Expression of a secretory protein C1qTNF6, a C1qTNF family member, in hepatocellular carcinoma. Analytical cellular pathology (Amsterdam) 2011, 34(3):113121.

21. O'Hanlon DM, Lynch J, Cormican M, Given HF: The acute phase response in breast carcinoma. Anticancer research 2002, 22(2b):1289-1293.

22. Gabay C, Kushner I: Acute-phase proteins and other systemic responses to inflammation. The New England journal of medicine 1999, 340(6):448-454. 
23. Allin $\mathrm{KH}$, Nordestgaard BG: Elevated C-reactive protein in the diagnosis, prognosis, and cause of cancer. Critical reviews in clinical laboratory sciences 2011, 48(4):155-170.

24. Ray K, Ujvari B, Ramana V, Donald J: Cross-talk between EGFR and IL- 6 drives oncogenic signaling and offers therapeutic opportunities in cancer. Cytokine \& growth factor reviews 2018, 41:18-27.

25. Shen ZT, Sigalov AB: Novel TREM-1 Inhibitors Attenuate Tumor Growth and Prolong Survival in Experimental Pancreatic Cancer. Molecular pharmaceutics 2017, 14(12):4572-4582.

26. Tran TH, Tran TTP, Truong DH, Nguyen HT, Pham TT, Yong CS, Kim JO: Toll-like receptor-targeted particles: A paradigm to manipulate the tumor microenvironment for cancer immunotherapy. Acta biomaterialia 2019, 94:82-96.

27. Zhao Z, Bo Z, Gong W, Guo Y: Inhibitor of Differentiation 1 (Id1) in Cancer and Cancer Therapy. International journal of medical sciences 2020, 17(8):995-1005.

28. Roschger C, Cabrele C: The Id-protein family in developmental and cancer-associated pathways. Cell communication and signaling: CCS 2017, 15(1):7.

29. Cheng YJ, Tsai JW, Hsieh KC, Yang YC, Chen YJ, Huang MS, Yuan SS: Id1 promotes lung cancer cell proliferation and tumor growth through Akt-related pathway. Cancer letters 2011, 307(2):191-199.

30. Han J, Flemington C, Houghton AB, Gu Z, Zambetti GP, Lutz RJ, Zhu L, Chittenden T: Expression of bbc3, a pro-apoptotic $\mathrm{BH} 3-$ only gene, is regulated by diverse cell death and survival signals. Proceedings of the National Academy of Sciences of the United States of America 2001, 98(20):11318-11323.

31. Wang S, Hou P, Pan W, He W, He DC, Wang H, He H: DDIT3 targets innate immunity via the DDIT3OTUD1-MAVS pathway to promote BVDV replication. Journal of virology 2020.

32. Liu H, Tian Z, Guo Y, Liu X, Ma Y, Du X, Wang R, Zhang S, Shi L, Guo H et al: Microcystin-leucine arginine exposure contributes to apoptosis and follicular atresia in mice ovaries by endoplasmic reticulum stress-upregulated Ddit3. The Science of the total environment 2021, 756:144070.

33. Tabas I, Ron D: Integrating the mechanisms of apoptosis induced by endoplasmic reticulum stress. Nature cell biology 2011, 13(3):184-190.

34. Li Y, Guo Y, Tang J, Jiang J, Chen Z: New insights into the roles of CHOP-induced apoptosis in ER stress. Acta biochimica et biophysica Sinica 2015, 47(2):146-147.

\section{Figures}


Fig. 1
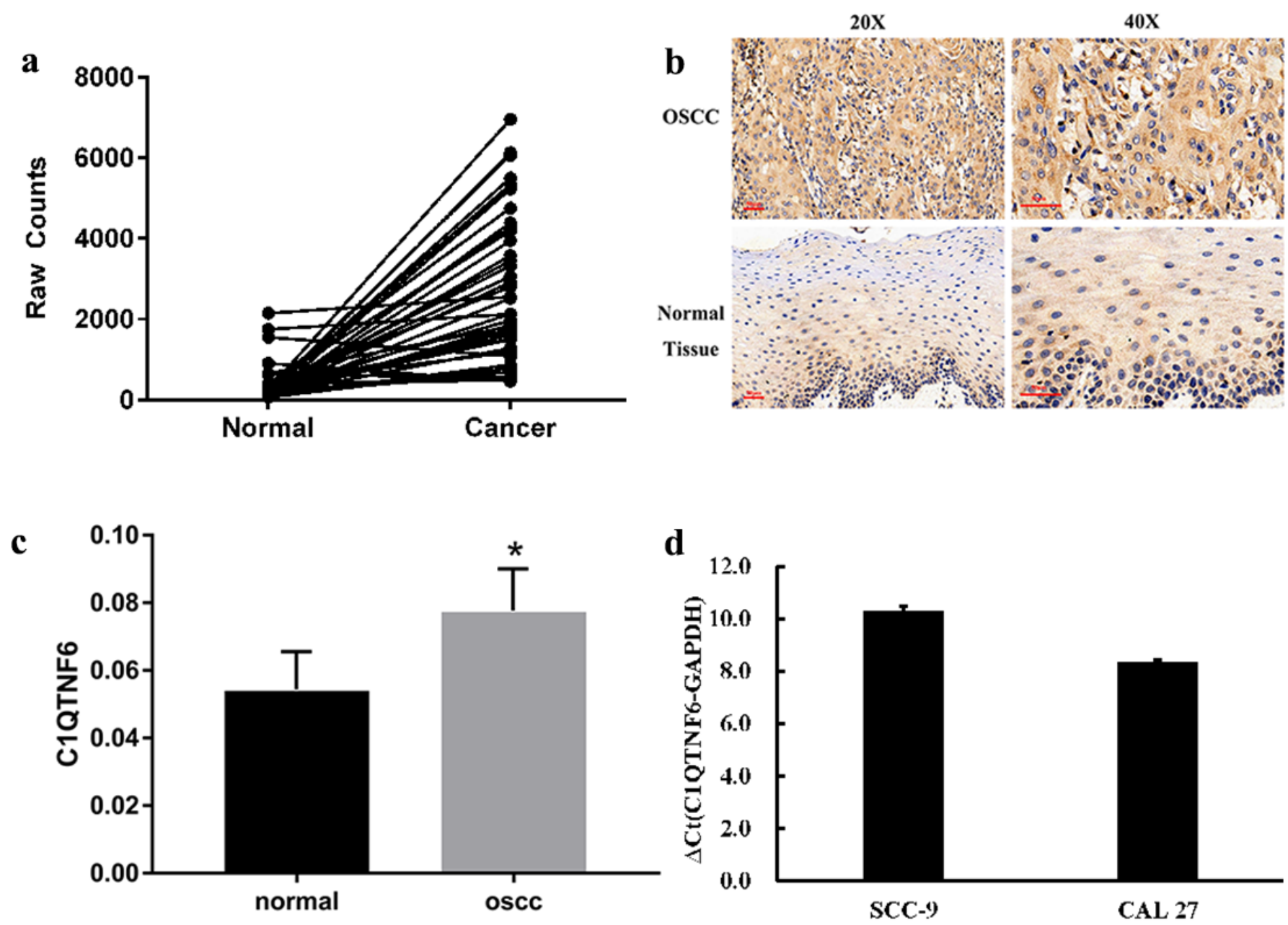

Figure 1

C1QTNF6 is high expressed in OSCC tissue and cell lines. a Analysis of data from The Cancer Genome Atlas (TCGA) showed that C1QTNF6 was significantly highly expressed in OSCC tissue compared to normal tissue. b\&c Immunohistochemical staining showed that the expression of C1QTNF6 in human OSCC tissues was higher than that in normal tissues. $d$ Quantitative real-time PCR demonstrated the high levels of C1QTNF6 mRNA in Cal-27 and SCC-9. 


\section{Fig. 2}
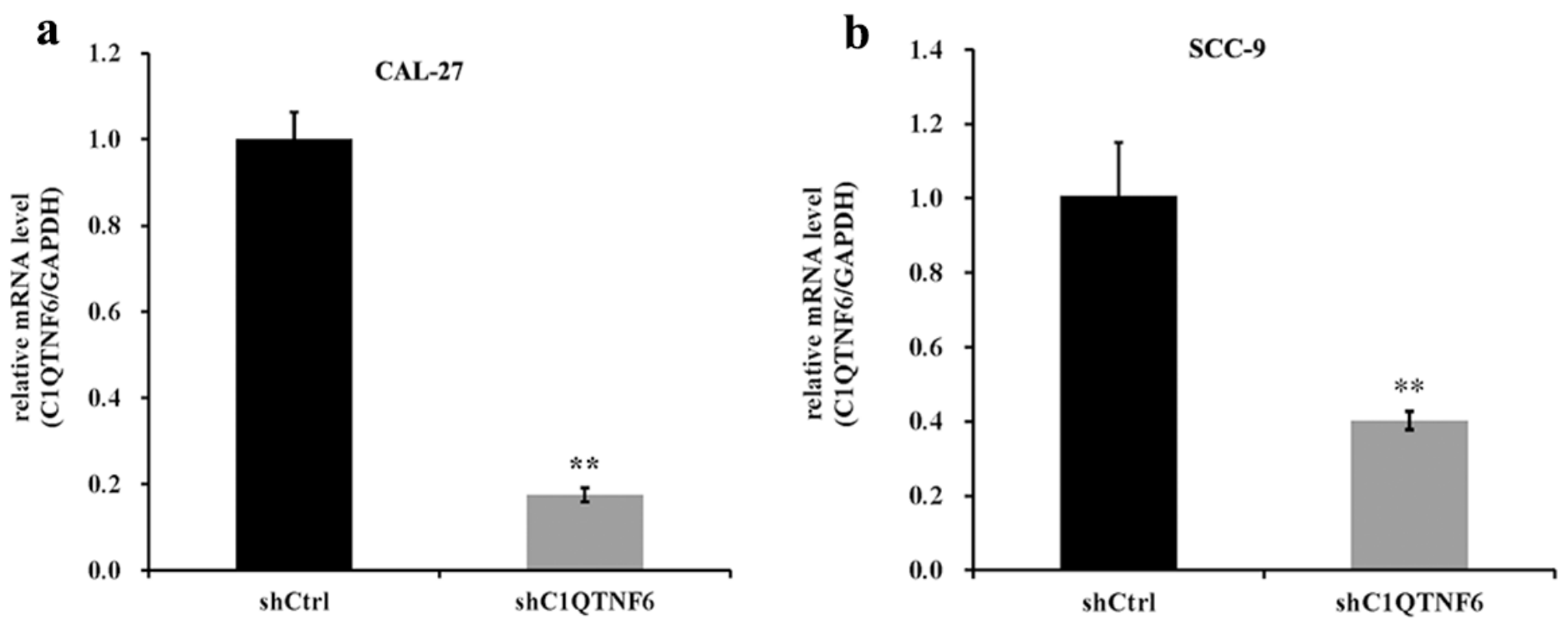

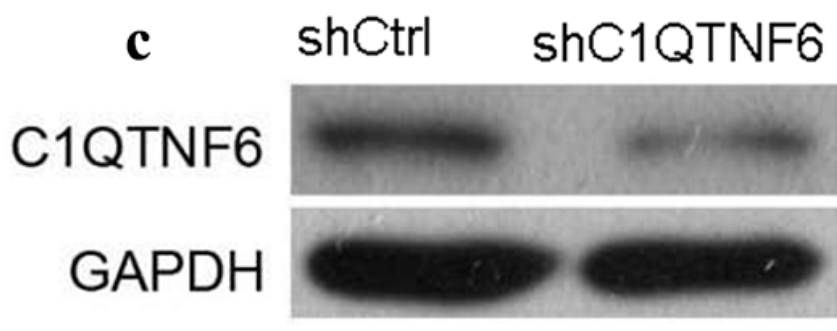

CAL-27

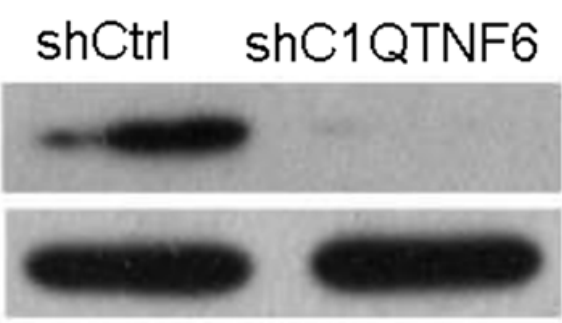

SCC-9

\section{Figure 2}

The Efficiency of C1QTNF6 knockdown in OSCC cell lines. a qRT-PCR analysis of siRNA-knockdown efficiency. Data are presented as the mean \pm SEM $(n=3), * * P<0.01$. b Western blot of C1QTNF6 expression in OSCC cells transinfected with LV-C1QTNF6-shRNA or LV-Ctrl-shRNA. GAPDH was used as a loading control. 
Fig. 3
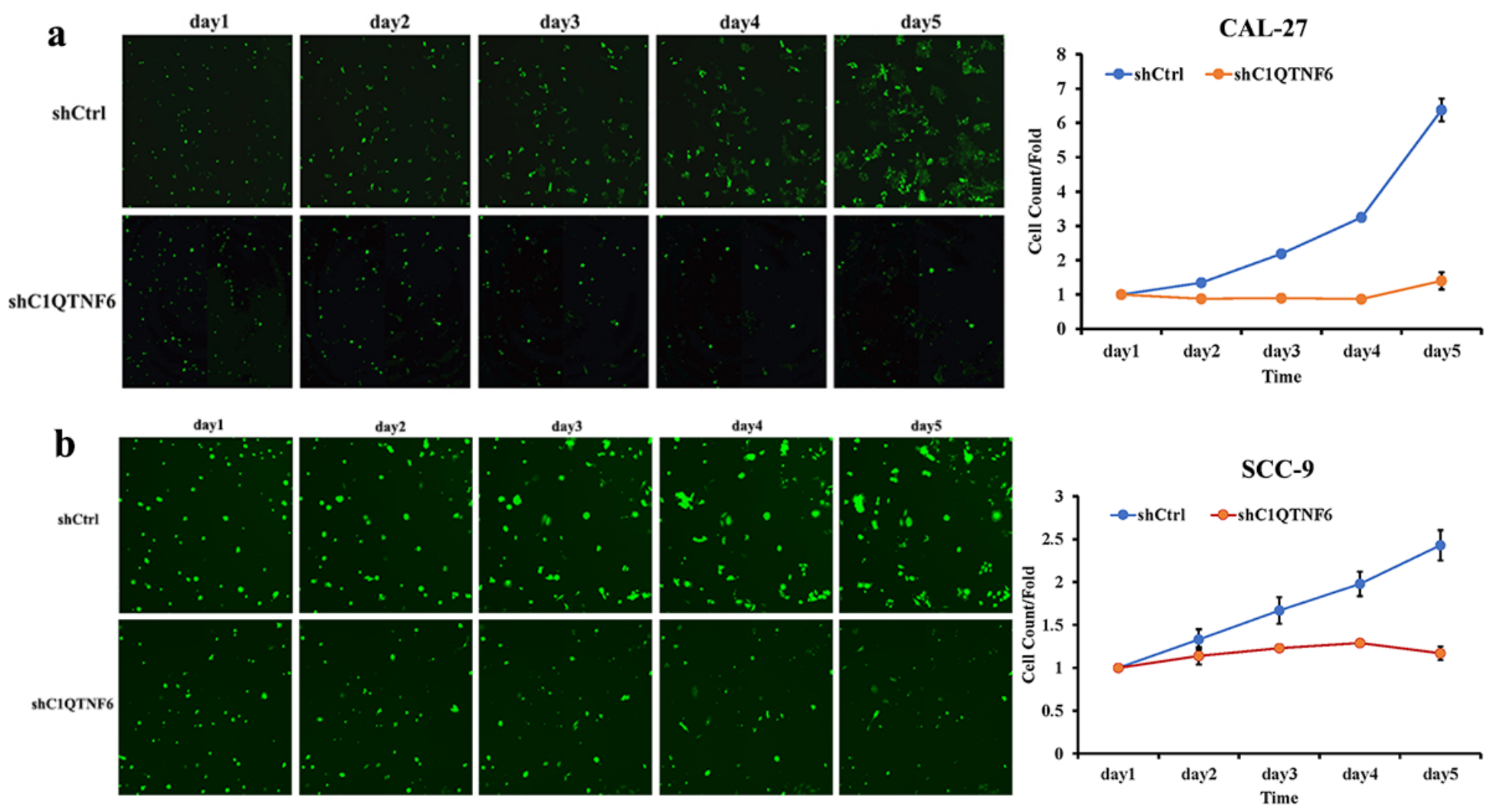

c
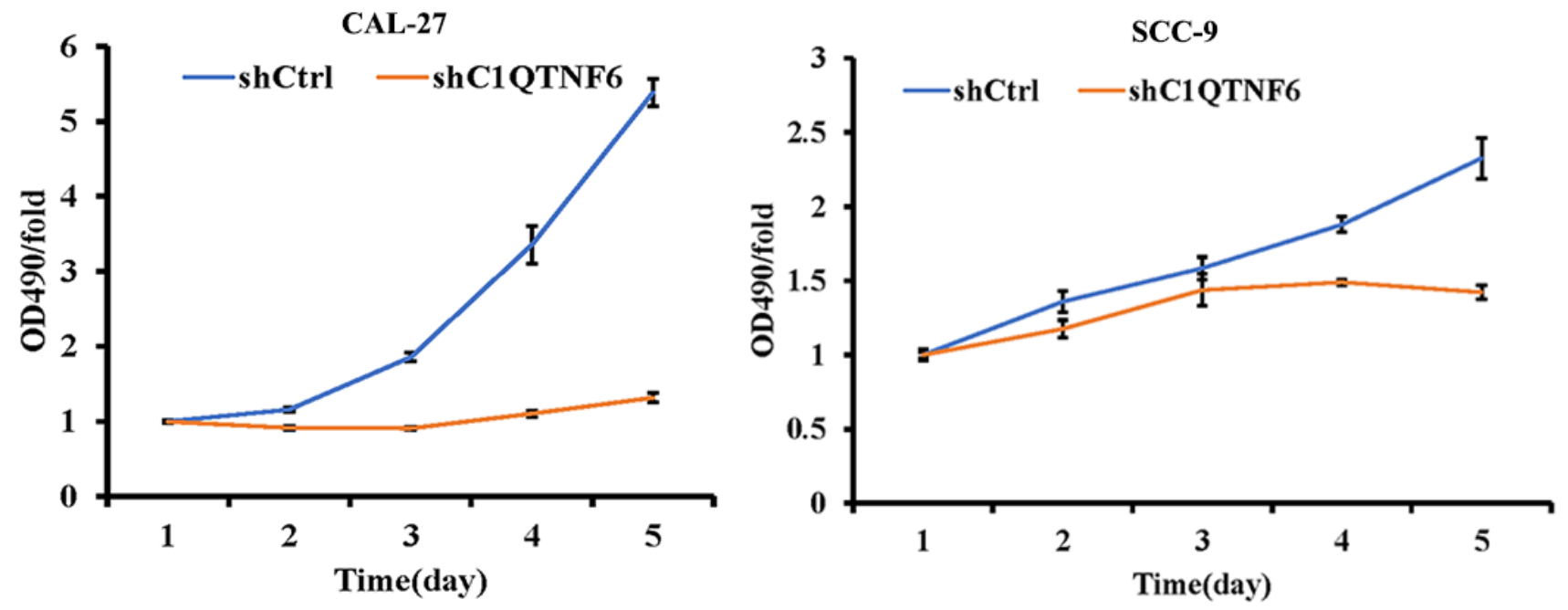

\section{Figure 3}

Knockdown of C1QTNF6 inhibits cell growth and proliferation. a\&b. Cell growth was measured by Celigo assay for five days. The images of fluorescence intensity were shown in Cal-27 cells (a) and SCC-9 cells (b) (magnification, x200). Cell growth curve of the siC1QTNF6 and shCtrl cells were displayed by cell number fold from days 1 to 5 . c The cell proliferation of Cal-27 cells (right panel) and SCC-9 cells (left panel) after Ctrl-shRNA or C1QTNF6-shRNA transfection were measured by the MTT assay. 
Fig. 4

CAL-27

SCC-9
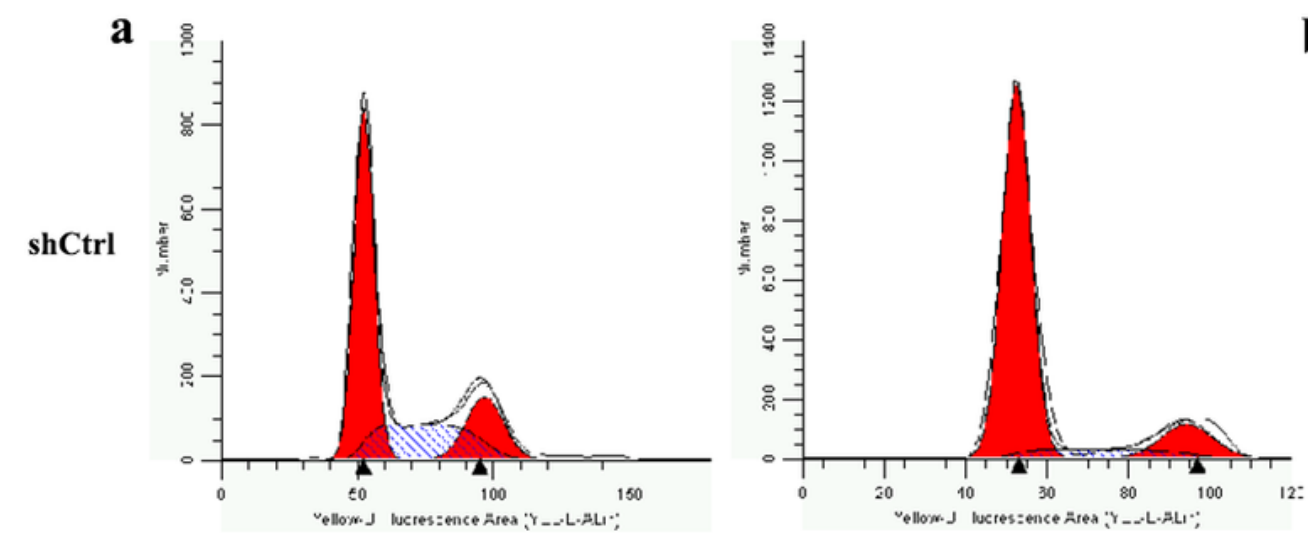

b
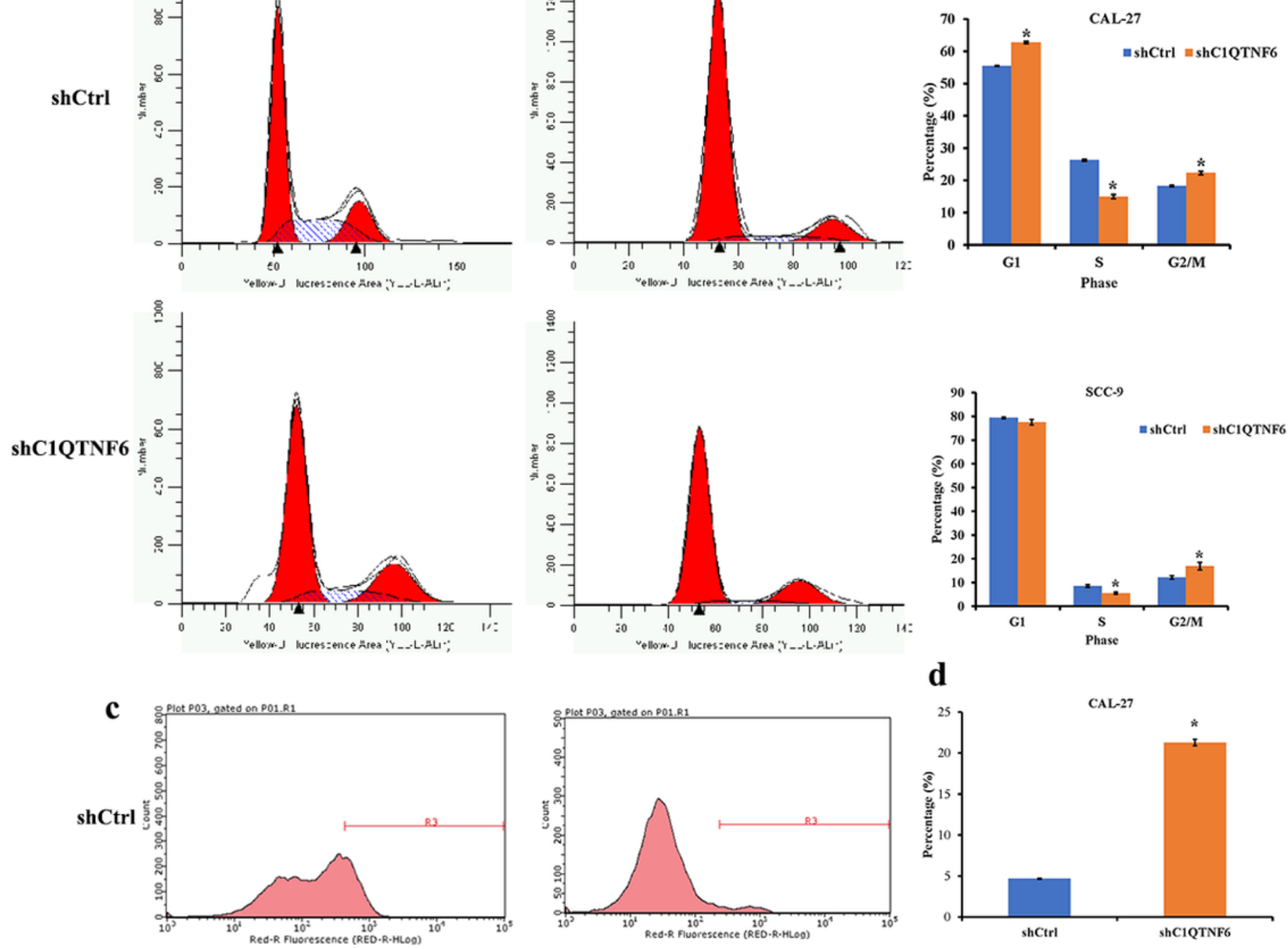

\section{d}
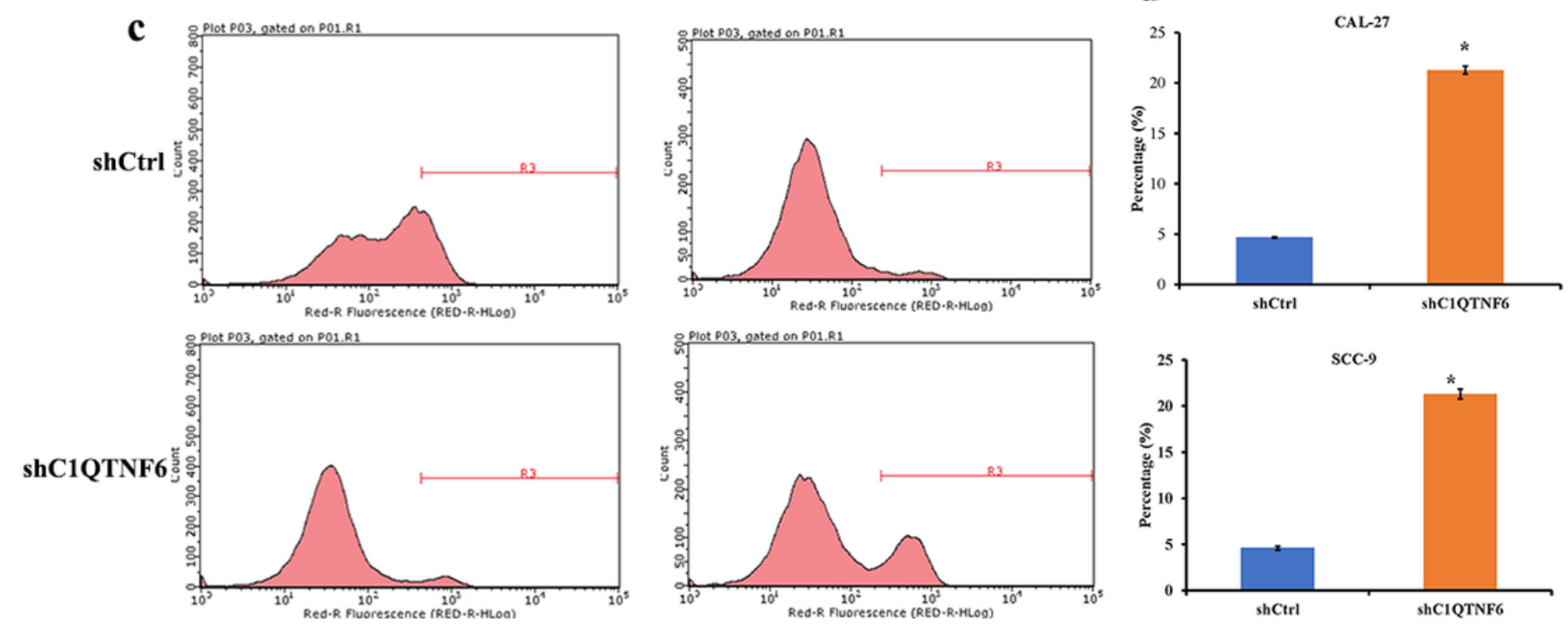

\section{Figure 4}

Knockdown of C1QTNF6 induces cell cycle arrest and apoptosis. Cell cycle distribution of Ctrl-shRNA and C1QTNF6-shRNA transfected cells was examined using PI staining, and analyzed by flow cytometry. a Representative images of PI staining of indicated cells. b The percentage of cells in the G0/G1, S, and $\mathrm{G} 2 / \mathrm{M}$ phases was summarized. Data represented the mean $\pm \mathrm{SD}$ of three independent experiments. * $\mathrm{P}$ $<0.05$. Apoptosis was assessed using Annexin-V staining. c Representative images of Annexin V-APC 
staining were shown. $d$ The percentage of cells in apoptosis were summarized. The rate of apoptosis was represented as mean \pm SD. $* P<0.05$

\section{Fig. 5}

a

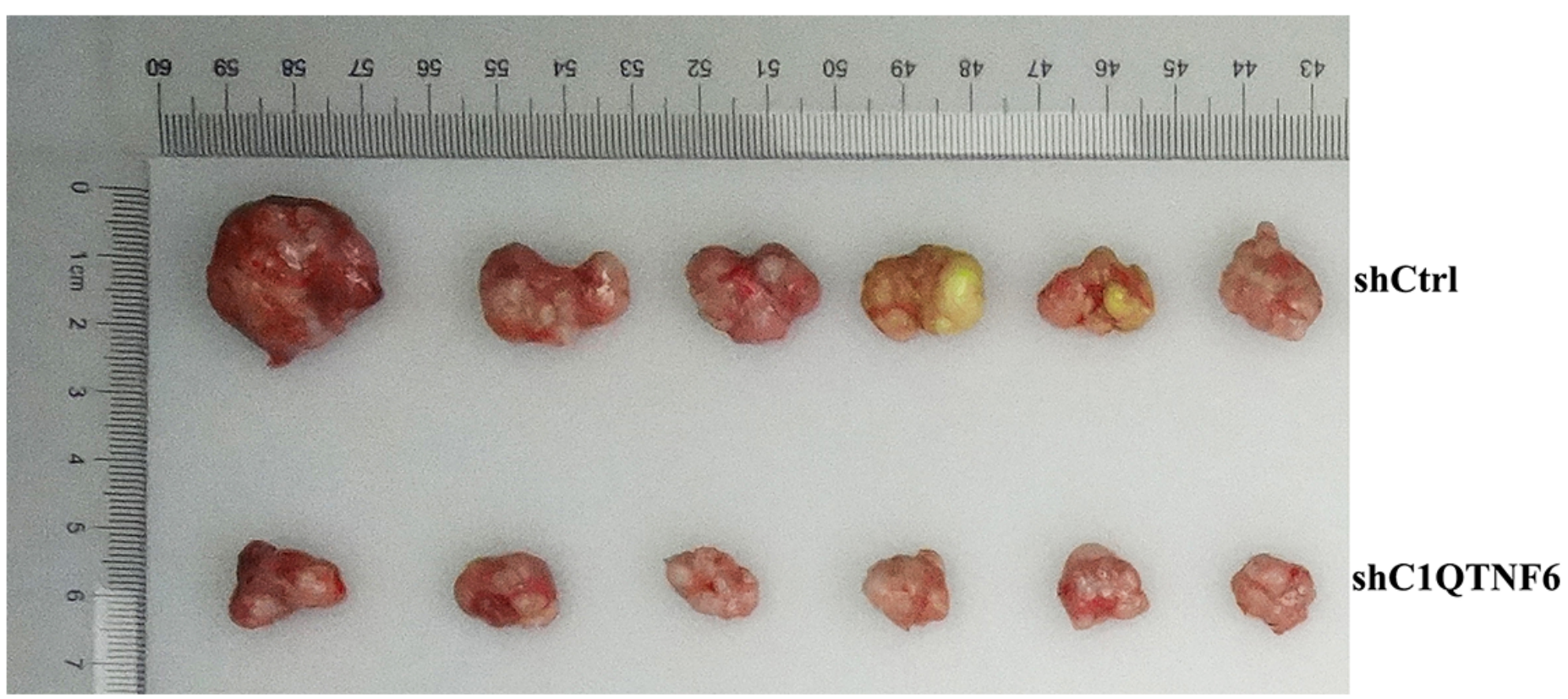

b

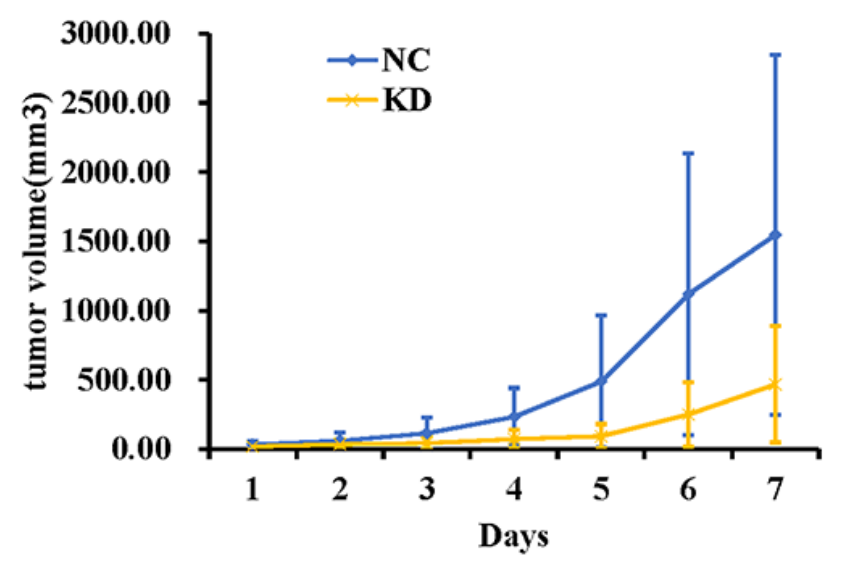

c

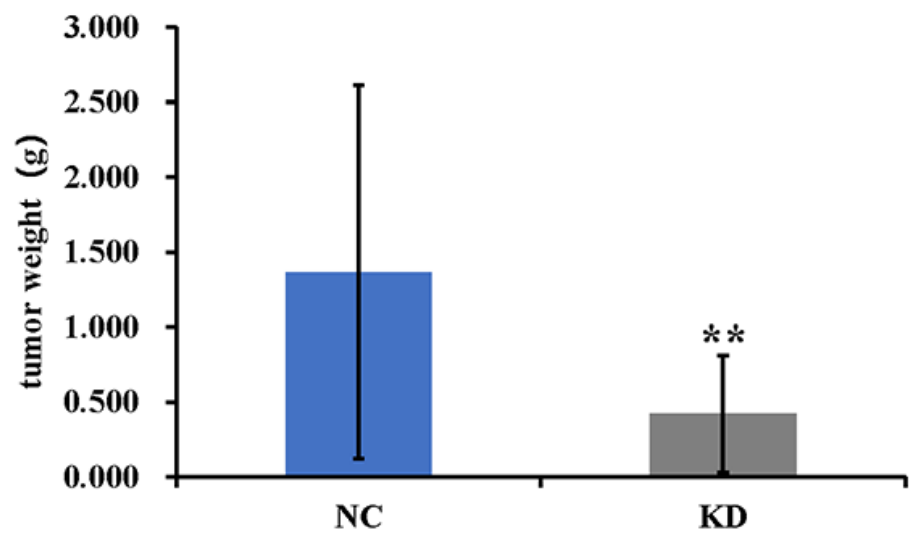

\section{Figure 5}

Knockdown of C1QTNF6 suppresses tumor growth in vivo. a\&b. Xenograft models nu/nu mice were generated with Ctrl-shRNA $(n=10)$ and C1QTNF6-shRNA $(n=10)$ transfected cells. Tumor volume was measured 7 times for 35 days. c Tumor weight in SCID mice inoculated with Ctrl-shRNA or C1QTNF6shRNA transfected cells was summarized. Data represented the mean $\pm S D(n=10) .\left({ }^{\star} P<0.05\right)$. 
Fig. 6
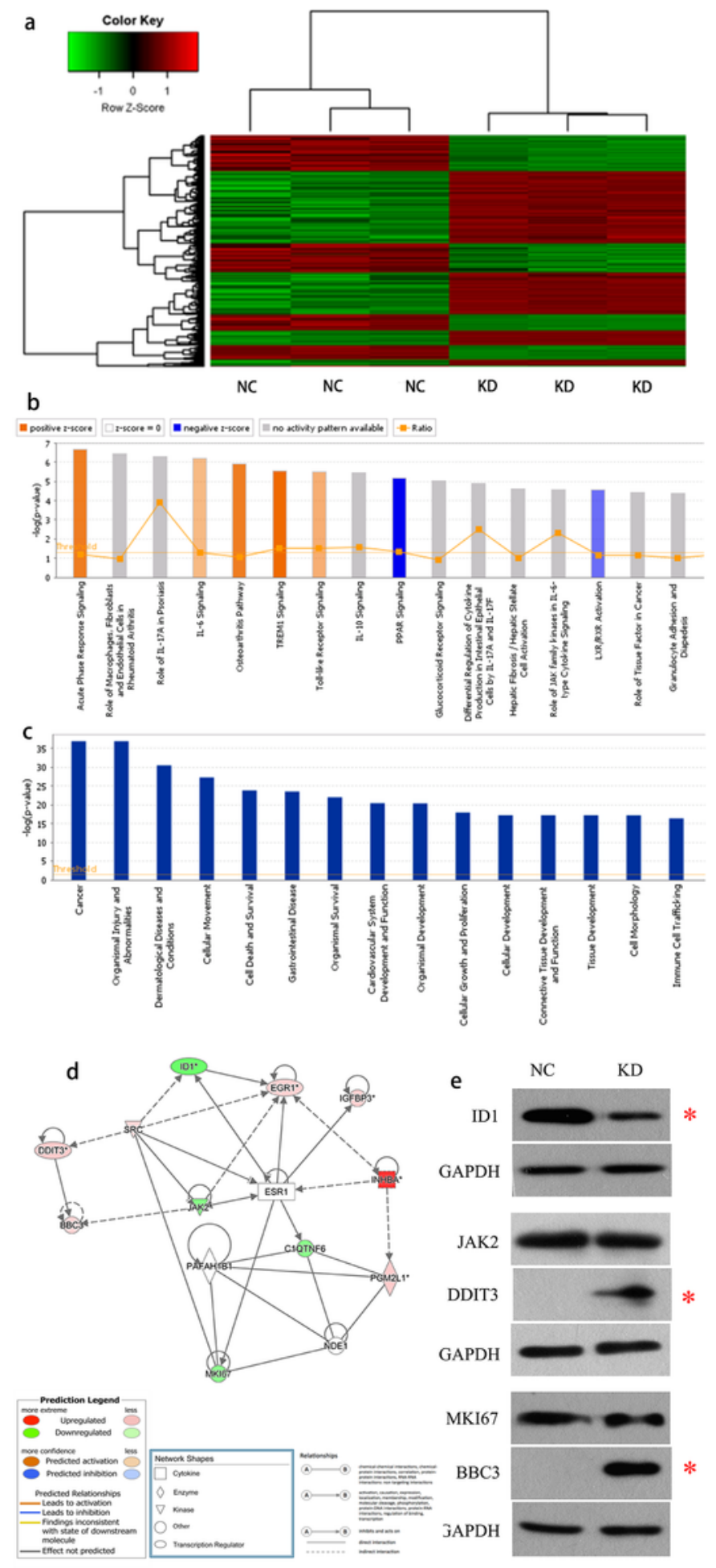

\section{Figure 6}

The knockdown of C1QTNF6 activated Acute Phase Response signaling pathway then targeted ID1, BBC3, DDIT3. a Hierarchical Clustering analysis heat map of DEGs, red indicates upregulation, green indicates downregulation, black means no obvious difference, gray means the gene not detected. $b$ Signaling pathways enriched among differentially expressed genes. The Y-axis represents the -log (10) P value for enrichment, with the threshold drawn at $P=0.05$. c Showed the significant enrichment of 
differential genes in diseases and functions. The abscissa is the disease or function name, and the ordinate is the significance level of enrichment (negative logarithmic transformation with base 10). $d$ Interaction network of several molecules closely connected to C1QTNF6. e Western blot verified the express changes. 